\title{
Minimal Polynomial Algorithms for Finite Sequences
}

\author{
Graham H. Norton \\ Dept. Mathematics, University of Queensland \\ Brisbane, Queensland 4072, Australia*
}

15 March, 2010

\begin{abstract}
We show that a straightforward rewrite of a known minimal polynomial algorithm yields a simpler version of a recent algorithm of A. Salagean.
\end{abstract}

Keywords: Berlekamp-Massey algorithm, characteristic polynomial, finite sequence, minimal polynomial.

\section{Introduction}

Let $K$ be a field, $n \geq 1$ and $s=\left(s_{1}, \ldots, s_{n}\right)$ be a finite sequence over $K$. The BerlekampMassey (BM) algorithm computes an LFSR of shortest length $L$ and a feedback polynomial $F \in K[x]$ generating $s$, vacuously if $L=n[1]$.

We begin with the approach and basics of [2]. Multiplication makes Laurent series in $x^{-1}$ into a $K[x]$-module and power series with non-zero annihilator ideal correspond to linear recurring sequences: they have a non-zero 'characteristic polynomial' (c.p.) [2, Section 2]. For finite sequences, we work with Laurent polynomials and $C \in K[x]$ is a c.p. of $s$ if

$$
C_{0} \cdot s_{j-d}+\cdots+C_{d} \cdot s_{j}=0
$$

for $d+1 \leq j \leq n$, where $d=\operatorname{deg}(C) \geq 0$ [2, Definition 2.7, Proposition 2.8]. Any $C$ with $d \geq n$ is vacuously a c.p. of $s$.

A c.p. $C$ of $s$ is a minimal polynomial of $s$ if $\operatorname{deg}(C)=\min \{\operatorname{deg}(D): D$ is a c.p. of $s\}$ and the linear complexity of $s$ is the degree of any minimal polynomial of $s$ [2, Definition 3.1], [3, Definition 2.2]. For example, $D=x^{L-\operatorname{deg}(F)} F^{*}$ is a c.p. of $s-$ as usual, $F^{*}$ is the reciprocal of $F$ - and $D$ is a minimal polynomial of $s$ since $D^{*}=F$ and $\operatorname{deg}(D)=L$.

\footnotetext{
*e-mail: ghn@maths.uq.edu.au
} 
As far as we know, Algorithm 4.2 of [2] (Algorithm 3.1 below) was the first algorithm to compute a minimal polynomial of $s$ iteratively. In fact, it is valid for finite sequences over a commutative unital integral domain.

Algorithm 2.2 of [4] also computes a minimal polynomial of $s$. We show that these two algorithms are closely related: a straightforward rewrite of the former using the notation of [4] yields the latter, except that we initialise a polynomial to 0 instead of 1 . Further, the rewrite uses fewer variables and is simpler. See also Remark 3.2 (iv).

We note that [2] and [3] (an expository version of [2]) were referred to in [5, Introduction].

\section{The Inductive Construction}

The whole process of Algorithm 3.1 is best explained in terms of the inductive construction of a minimal polynomial of $s$ which was derived from first principles in [2].

\subsection{The Naive Version}

A natural choice for $C^{(1)}$ is 1 if $s_{1}=0$ and $x$ otherwise; $C^{(1)}$ is certainly a c.p. of minimal degree. Now assume inductively that $2 \leq i \leq n$ and we have c.p.'s $C^{(j)}$ for $\left(s_{1}, \ldots, s_{j}\right)$ where $1 \leq j \leq i-1$. From Equation (1),$C^{(i-1)}$ is a c.p. of $\left(s_{1}, \ldots, s_{i}\right)$ if and only if the discrepancy

$$
c_{i-1}=\mathrm{d}\left(C^{(i-1)}\right)=\sum_{j=0}^{d_{i-1}} C_{j}^{(i-1)} \cdot s_{j+i-d_{i-1}} \in K
$$

is zero, where $d_{i-1}=\operatorname{deg}\left(C^{(i-1)}\right)$ [2, Definition 2.10], cf. [1, Equation (10)]. If $c_{i-1}=0$ then clearly $d_{i-1}$ is minimal. But if $c_{i-1} \neq 0$, a new c.p. is needed. We use an index $a_{i-1}$ suggested by [1, Equation (11)]

$$
a=a_{i-1}=\max _{1 \leq j \leq i-2}\left\{j: d_{j}<d_{i-1}\right\}
$$

to index a previous c.p. [2, Definition 3.12]. The exponent $e_{i-1}=2 d_{i-1}-i$ and $C^{(i)}$ are from [2, Proposition 4.1]:

$$
C^{(i)}=\left\{\begin{array}{l}
c_{a} \cdot C^{(i-1)}-c_{i-1} \cdot x^{+e} C^{(a)} \text { if } e=e_{i-1} \geq 0 \\
c_{a} \cdot x^{-e} C^{(i-1)}-c_{i-1} \cdot C^{(a)} \text { otherwise. }
\end{array}\right.
$$

Now $a=a_{i-1}$ and Equation (3) require $i \geq 3$ and $d_{i-1}>d_{1}$. For $i \geq 2$ and $d_{i-1}=d_{1}$, we complete our construction by

$$
C^{(i)}= \begin{cases}x^{i} & \text { if } s_{1}=0 \\ s_{1} \cdot x^{i-2} C^{(i-1)}-c_{i-1} & \text { otherwise }\end{cases}
$$


The case $s_{1}=0$ occurs when $s$ has $i-1 \geq 1$ leading zeroes.

Part (i) of the following proposition is an analogue of [1, Equation (15)]. For Part (ii), see [3, Propositions 4.3, 4.5].

Proposition 2.1 For $2 \leq i \leq n$, if $c_{i-1} \neq 0$ then (i) $d_{i}=\max \left\{d_{i-1}, i-d_{i-1}\right\}$ and (ii) $C^{(i)}$ is a c.p. of $\left(s_{1}, \ldots, s_{i}\right)$.

An analogue of [1, Lemma 1] establishes minimality.

Lemma 2.2 ([2, Theorems 3.8, 3.13] or [3, Lemma 5.2]) Let $2 \leq i \leq n, f$ be a c.p. of $\left(s_{1}, \ldots, s_{i-1}\right)$ and $\mathrm{d}(f) \neq 0$. If $g$ is a c.p. of $\left(s_{1}, \ldots, s_{i}\right)$, then $\operatorname{deg}(g) \geq i-\operatorname{deg}(f)$.

We now have a naive inductive construction for a minimal polynomial of $s$ and illustrate it with two binary examples.

Example 2.3 Consider the subsequence $s=(0,1,1,0)$ of [4, Table I]. We have $C^{(1)}=1$ as $s_{1}=0$ and $c_{1}=s_{2} \neq 0$. As $i<3$, we apply Equation (4): $C^{(2)}=x^{2}$ (there is one leading zero). Now $i=3, c_{2}=s_{3} \neq 0$ and $d_{2}=2>d_{1}=0$, so that $a_{2}=1$. Equation (3) applies with exponent $e_{2}=2 \cdot 2-3=1$ giving $C^{(3)}=C^{(2)}+x^{1} C^{(1)}=x^{2}+x$. Finally, $c_{3}=s_{4}+s_{3} \neq 0$ and $d_{3}=2>d_{1}=0$, so that $a_{3}=a_{2}=1$. Equation (3) applies again with exponent $e_{3}=2 \cdot 2-4=0$ and $C^{(4)}=C^{(3)}+x^{0} C^{(1)}=x^{2}+x+1$.

Example 2.4 Let $s=(1,1,0,0)$. Clearly $C^{(1)}=x$ and $c_{1}=s_{2} \neq 0$, so $C^{(2)}=$ $x^{0} C^{(1)}+1=x+1$ from Equation (41). Next, $c_{2}=s_{3}+s_{2} \neq 0$ and Equation (44) obtains as $d_{2}=d_{1}=1$, giving $C^{(3)}=x^{1} C^{(2)}+c_{2}=x^{2}+x+1$. For $i=4, c_{3}=s_{4}+s_{3}+s_{2} \neq 0$, $d_{3}=2>d_{1}=1$ and $a_{3}=2$. Equation (3) with exponent $e_{3}=2 \cdot 2-4=0$ gives $C^{(4)}=C^{(3)}+x^{0} C^{(2)}=x^{2}$.

\subsection{The Refined Version}

The naive construction can be refined in three ways. Firstly, by noting that if $e_{i-1} \geq 0$ then $a_{i}=a_{i-1}$ since $d_{i-1} \geq i-d_{i-1}$ i.e. $d_{i}=d_{i-1}$ by Proposition 2.1. But if $e_{i-1}<0$, $d_{i}=i-d_{i-1}>d_{i-1}$ and $a_{i}=i-1$.

Secondly, we update $e_{i-1}=2 d_{i-1}-i$ (and so avoid using $d_{i-1}$ ) as follows: it is trivial that $e_{i}=e_{i-1}-1$ if $c_{i-1}=0$ and easy to check that $e_{i}=\left|e_{i-1}\right|-1$ if $c_{i-1} \neq 0$.

Thirdly, we change the inductive basis by introducing artificial values $C^{(0)}=1$ (which is only a c.p. if $s_{1}=0$ ), $C^{\left(a_{0}\right)}=0$ (which is not a c.p. by definition) and $c_{a_{0}}=1$ (which is not a discrepancy). For definiteness, we take $a_{0}=-1$. Then remarkably (i) $e_{0}=-1$ (ii) Equation (3) accomodates all three cases and (iii) the updating of $a_{i-1}$ and $e_{i-1}$ remains valid. We state this formally.

Theorem $2.5([2])$ Put $a_{0}=e_{0}=-1, C^{(-1)}=0, c_{-1}=1$ and $C^{(0)}=1$. For $1 \leq i \leq n$, define $C^{(i)}, a_{i}, e_{i}$ by

(i) if $c_{i-1}=0, C^{(i)}=C^{(i-1)}, a_{i}=a_{i-1}$ and $e_{i}=e_{i-1}-1$ 
(ii) if $c_{i-1} \neq 0$ let $C^{(i)}$ be as in Equation (3), $a_{i}=a_{i-1}$ if $e_{i-1} \geq 0, a_{i}=i-1$ if $e_{i-1}<0$ and $e_{i}=\left|e_{i-1}\right|-1$.

Then for $1 \leq i \leq n, C^{(i)}$ is a minimal polynomial of $\left(s_{1}, \ldots, s_{i}\right)$ and $\operatorname{deg}\left(C^{(i)}\right)=\frac{e_{i}+i+1}{2}$.

The BM algorithm decodes Reed-Solomon, Goppa and negacyclic codes [6], [7] and has been extended to multiple sequences [8]. For similar applications and extensions of Theorem 2.5, see [3, Section 8], [9].

\section{The Algorithm}

We deduce Algorithm 3.1 from Theorem 2.5. Firstly, we can dispense with the indices $a_{i}$ if we define $B^{(i)}=C^{\left(a_{i}\right)}$ and scalars $b_{i}=\mathrm{d}\left(C^{\left(a_{i}\right)}\right)$ for $0 \leq i \leq n-1$ provided we update $B^{(i-1)}$ and $b_{i-1}$ when $e_{i-1}<0$. Secondly, we replace $d_{i-1}$ by $\frac{e_{i-1}+i}{2}$ in $c_{i-1}$. Finally, since only current values are used we can suppress all indices - provided we keep a copy $T$ of $C^{(i-1)}$ to update $B^{(i-1)}$ when $e_{i-1}<0$.

Algorithm 3.1 ([2, Algorithm 4.2] rewritten) Input: $n \geq 1$ and a sequence $s=\left(s_{1}, \ldots, s_{n}\right)$ over a field $K$. Output: $C$, a monic minimal polynomial for $s$. begin $B \leftarrow 0 ; b \leftarrow 1 ; C \leftarrow 1 ; e \leftarrow-1$;

for $i=1$ to $n$ do

$c \leftarrow \sum_{j=0}^{\frac{e+i}{2}} C_{j} \cdot s_{j+\frac{i-e}{2}} ;$

if $c \neq 0$ then if $e \geq 0$ then $C \leftarrow b \cdot C-c \cdot x^{e} B$;

else $T \leftarrow C ; e \leftarrow-e$;

$C \leftarrow b \cdot x^{e} C-c \cdot B$

$B \leftarrow T ; b \leftarrow c$; endif

endif

$e \leftarrow e-1$

endfor

return $(C / b)$ end.

Remark 3.2 We obtain [4, Algorithm 2.2] except that

(i) we could (but do not) make each $C$ monic

(ii) in the notation of [4, p. 4696], we do not keep track of ' $m$ ' or $\operatorname{deg}(B)$ to recompute $v=N-m-(\operatorname{deg}(C)-\operatorname{deg}(B))$ at each iteration (where $0 \leq N \leq n-1)$

(iii) in fact, (a) $N$ corresponds to $i-1$ (b) if there are $N$ leading zeroes $v=N+1$ (c) if $\operatorname{deg}(C)>\operatorname{deg}(B)$ then $\operatorname{deg}(C)=m+1-\operatorname{deg}(B)$ and $v=N-m-(\operatorname{deg}(C)-\operatorname{deg}(B))=$ $N+1-2 \operatorname{deg}(C)$; thus $v$ corresponds to $-e$

(iv) $B^{(0)}=0$ and some initial minimal polynomials will differ; with $B^{(0)}=C^{(-1)}=1$, $C^{(i)}=x^{i}-c_{i-1}$ when there are $i-1 \geq 0$ leading zeroes, Theorem 2.5 remains valid, the 
algorithms are equivalent and their outputs are identical.

\section{Remarks 3.3 (added in proof)}

(i) Theorem 2.4 of [4] on the set of minimal polynomials of $s$ is an immediate consequence of [2, Theorem 4.16]: simply replace $d$ in Theorem 4.16, loc. cit. by $-v=$ $\operatorname{deg}(C)-\operatorname{deg}(B)-(n-m)$ and take first components.

(ii) In [10, we calculate the monic reciprocals of the minimal polynomials of Theorem 2.5. This readily yields an algorithm similar to the BM algorithm, except that we do not calculate the 'lengths' $L_{i}$; we update the exponents as above and obtain $L_{n}$ as $\frac{e_{n}+n+1}{2}$. This article also contains proofs for the results of Section 2 using similar notation.

The author thanks the anonymous reviewers for their helpful comments and suggestions.

\section{References}

[1] J. L. Massey. Shift-register synthesis and BCH decoding. IEEE Trans. Inform. Theory, 15:122-127, 1969.

[2] Graham H. Norton. On the minimal realizations of a finite sequence. J. Symbolic Computation, 20:93-115, 1995.

[3] Graham H. Norton. On shortest linear recurrences. J. Symbolic Computation, 27:323$347,1999$.

[4] A. Salagean. An Algorithm for Computing Minimal Bidirectional Linear Recurrence Relations. IEEE Trans. Inform. Theory, 55:4695-4700, 2009.

[5] Graham H. Norton and A. Salagean. On the key equation over a commutative ring. Designs, Codes and Cryptography, 20:125-141, 2000.

[6] E.R. Berlekamp, Algebraic Coding Theory, McGraw-Hill Series in Systems Science, New York-Toronto, 1968.

[7] N.J. Patterson. The algebraic decoding of Goppa codes IEEE Trans. Inform. Theory, 21:203-207, 1975.

[8] G.L. Feng and K.K. Tzeng. A generalization of the Berlekamp-Massey algorithm for multisequence shift register sequence synthesis with applications to decoding cyclic codes, IEEE Trans. Inform. Theory, 37:1274-1287,1991.

[9] Graham H. Norton. Some decoding applications of minimal realization, In (C. Boyd Ed.) Cryptography and Coding, Lecture Notes in Computer Science, Springer, 1025:5362, 1995. 
[10] Graham H. Norton. The Berlekamp-Massey Algorithm via Minimal Polynomials. http://arXiv.org, 1001.1597. 